\title{
The Real Rank of CCR $C^{*}$-Algebras
}

\author{
TAKAHIRo SUdo \\ Department of Mathematical Sciences, Faculty of Science, University of the \\ Ryukyus, Nishihara, Okinawa 903-0213, Japan \\ e-mail: sudo@math.u-ryukyu.ac.jp
}

Abstract. We estimate the real rank of CCR $C^{*}$-algebras under some assumptions. As applications we determine the real rank of the reduced group $C^{*}$-algebras of non-compact connected, semi-simple and reductive Lie groups and that of the group $C^{*}$-algebras of connected nilpotent Lie groups.

\section{Introduction}

The real rank for $C^{*}$-algebras was introduced by Brown and Pedersen [3]. By definition, we say that a unital $C^{*}$-algebra $\mathfrak{A}$ has the real rank $n=\operatorname{RR}(\mathfrak{A})$ if $n$ is the smallest non-negative integer such that for any $\varepsilon>0$ given, any self-adjoint element $\left(a_{j}\right)_{j=1}^{n+1} \in \mathfrak{A}^{n+1}$ with $a_{j}=a_{j}^{*}$ is approximated by a self-adjoint element $\left(b_{j}\right)_{j=1}^{n+1} \in \mathfrak{A}^{n+1}$ with $b_{j}=b_{j}^{*}$ such that $\left\|a_{j}-b_{j}\right\|<\varepsilon(1 \leq j \leq n+1)$ and $\sum_{j=1}^{n+1} b_{j}^{2}$ is invertible in $\mathfrak{A}$. For a non-unital $C^{*}$-algebra, its real rank is defined by that of its unitization by $C$. By definition, $\operatorname{RR}(\mathfrak{A}) \in\{0,1,2, \cdots, \infty\}$.

On the other hand, $\mathrm{CCR} C^{*}$-algebras are very well known in the $C^{*}$-algebra theory such as the representation theory and structure theory of $C^{*}$-algebras (or group $C^{*}$-algebras). Recall that a $C^{*}$-algebra $\mathfrak{A}$ is $\mathrm{CCR}$ (or liminary) if for any irreducible representation $\pi$ of $\mathfrak{A}$, the image $\pi(\mathfrak{A})$ is either isomorphic to a matrix algebra $M_{n}(C)$ over $C$ or to $K$ the $C^{*}$-algebra of compact operators on a separable infinite dimensional Hilbert space (Dixmier [4, Section 4.2] and Pedersen [11, Section $6.1])$.

However, it seems that the real rank of CCR $C^{*}$-algebras has been unknown. It is in part because it is difficult in general to compute the real rank of extensions of $C^{*}$-algebras. Thus as the first step we impose an assumption on CCR $C^{*}$-algebras that they have no finite dimensional irreducible representations. Then we can show below that those CCR $C^{*}$-algebras have real rank less than or equal to one by using their composition series and some results on the real rank for extensions of $C^{*}$-algebras (Nagisa, Osaka and Phillips [9]) and for tensor products of $C^{*}$-algebras with $K$ (Beggs and Evans [2]).

As applications, we show that the real rank of the reduced group $C^{*}$-algebras of

Received April 17, 2006.

2000 Mathematics Subject Classification: 46L05.

Key words and phrases: $\mathrm{C}^{*}$-algebra, real rank, group $C^{*}$-algebras. 
non-compact connected semi-simple Lie groups is one, and that of the reduced group $C^{*}$-algebras of non-compact connected reductive Lie groups is also one by using [13] in part for the structure of these group $C^{*}$-algebras. These results should be new and interesting. Compare them with the stable rank of those group $C^{*}$-algebras [13] (and see below). See also Kaniuth [7].

Moreover, following the same methods for the real rank of those $\mathrm{CCR} C^{*}$ algebras and using [2] for the real rank of tensor products of commutative $C^{*}$ algebras with $M_{n}(C)$ we show that the real rank of $\mathrm{CCR} C^{*}$-algebras which have no infinite dimensional irreducible representations can be estimated in terms of homogeneous subquotients with continuous trace.

Finally, we estimate the real rank of a CCR $C^{*}$-algebra that is decomposed into a closed ideal and a quotient that have no finite and no infinite dimensional irreducible representations respectively. As the final application, we show that the real rank of the group $C^{*}$-algebras of connected nilpotent Lie groups is equal to the dimension of the spaces of their 1-dimensional representations. For the stable rank of these group $C^{*}$-algebras, see [17] (and see below).

\section{The real rank of CCR $C^{*}$-algebras}

Theorem 2.1. Let $\mathfrak{A}$ be a CCR $C^{*}$-algebra. Suppose that $\mathfrak{A}$ has no finite dimensional irreducible representations. Then $\mathrm{RR}(\mathfrak{A}) \leq 1$.

Proof. Since $\mathfrak{A}$ is of type I, it has a composition series $\left(\mathfrak{I}_{j}\right)$ of essential closed ideals such that the union $\cup_{j} \mathfrak{I}_{j}$ is dense in $\mathfrak{A}$ and the subquotients $\mathfrak{I}_{j} / \mathfrak{I}_{j-1}$ have continuous trace and each $\mathfrak{I}_{j}$ is essential in $\mathfrak{I}_{j+1}([11$, Theorem 6.2 .11$]$, [4, Theorem 4.5.5]). Thus, the subquotients $\mathfrak{I}_{j} / \mathfrak{I}_{j-1}$ for $j \geq 1$ with $\mathfrak{I}_{0}=\{0\}$ have Hausdorff spectrums, and by the assumption they are regarded as continuous field $C^{*}$-algebras $\Gamma_{0}\left(X_{j},\{K\}\right)$ on their spectrums $X_{j}$ with fibers the constant $K$ consisting of (certain) continuous operator fields on $X_{j}$ vanishing at infinity ([4, Theorem 10.5.4]). Since the continuous field $C^{*}$-algebras $\Gamma_{0}\left(X_{j},\{K\}\right)$ are locally trivial ([4, Theorem 10.8.8]), they are inductive limits of the tensor products $C_{0}\left(U_{j k}\right) \otimes K$ for (certain) increasing open subsets $U_{j k}$ of $X_{j}$ with the unions $\cup_{k} U_{j k}=X_{j}$, where $C_{0}\left(U_{j k}\right)$ are the $C^{*}$-algebras of continuous functions on $U_{j k}$ vaninshing at infinity.

As the first step, we have the following exact sequence:

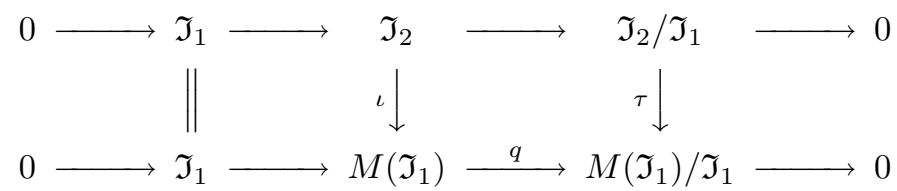

where $M\left(\mathfrak{I}_{1}\right)$ is the multiplier algebra of $\mathfrak{I}_{1}$, and $\mathfrak{I}_{2}$ is isomorphic to the pull back $M\left(\mathfrak{I}_{1}\right) \oplus_{q, \tau} \mathfrak{I}_{2} / \mathfrak{I}_{1}$ defined by $\left\{(x, y) \in M\left(\mathfrak{I}_{1}\right) \oplus \mathfrak{I}_{2} / \mathfrak{I}_{1} \mid q(x)=\tau(y)\right\}$, where $q$ is the canonical quotient map and $\tau$ is the Busby invariant associated with the extension, and $\iota$ is the canonical inclusion (cf. [19]). Since $\mathfrak{I}_{1} \cong \Gamma_{0}\left(X_{1},\{K\}\right)$, we have $M\left(\mathfrak{I}_{1}\right) \cong$ $\Gamma^{b}\left(X_{1},\{B\}\right)$ the $C^{*}$-algebra of a bounded continuous field on $X_{1}$ with fibers the 
constant $B$ the $C^{*}$-algebra of all bounded operators with the strict topology (cf. [1]). However, since $\mathfrak{I}_{2}$ is CCR and $\iota$ is injective from that $\mathfrak{I}_{1}$ is essential in $\mathfrak{I}_{2}$, we in fact that $\mathfrak{I}_{2}$ is embedded in $\Gamma^{b}\left(X_{1},\{K\}\right)$. Therefore, we have

$$
\mathfrak{I}_{2} \cong \Gamma^{b}\left(X_{1},\{K\}\right) \oplus_{q, \tau} \mathfrak{I}_{2} / \mathfrak{I}_{1} .
$$

Furthermore, we note that $\Gamma^{b}\left(X_{1},\{K\}\right)$ is stable, that is,

$$
\Gamma^{b}\left(X_{1},\{K\}\right) \cong \Gamma^{b}\left(X_{1},\{K\}\right) \otimes K,
$$

which follows from $\Gamma^{b}\left(X_{1},\{K\}\right) \otimes M_{n}(C) \cong \Gamma^{b}\left(X_{1},\left\{K \otimes M_{n}(C)\right\}\right) \cong \Gamma^{b}\left(X_{1},\{K\}\right)$ for any $n \geq 1$, and we can replace $M_{n}(C)$ with $K$. Note also that continuous fields with fibers $K$ are always locally trivial (see [4, Chapter 10]), and it is known that an inductive limit of stable $C^{*}$-algebras is also stable [6].

By using [9, Proposition 1.6] and [2, Proposition 3.3],

$$
\begin{aligned}
\operatorname{RR}\left(\mathfrak{I}_{2}\right) & \leq \max \left\{\operatorname{RR}\left(\Gamma^{b}\left(X_{1},\{K\}\right) \otimes K\right), \operatorname{RR}\left(\mathfrak{I}_{2} / \mathfrak{I}_{1}\right)\right\} \\
& \leq \max \left\{1, \operatorname{RR}\left(\mathfrak{I}_{2} / \mathfrak{I}_{1}\right)\right\}=1
\end{aligned}
$$

since $\mathfrak{I}_{2} / \mathfrak{I}_{1} \cong \lim _{\longrightarrow} C_{0}\left(U_{2 k}\right) \otimes K$ so that

$$
\left.\mathrm{RR}\left(\lim _{\longrightarrow} C_{0}\left(U_{2 k}\right) \otimes K\right) \leq \sup _{k} \operatorname{RR}\left(C_{0}\left(U_{2 k}\right) \otimes K\right)\right) \leq 1 .
$$

As the second step, we have the following commutative diagrams:

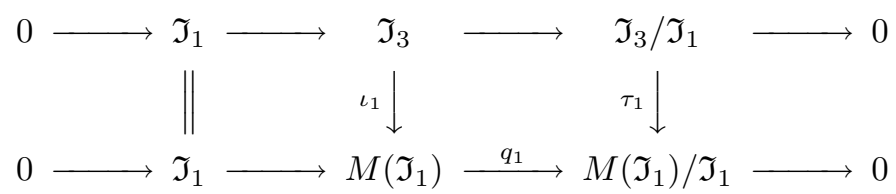

and

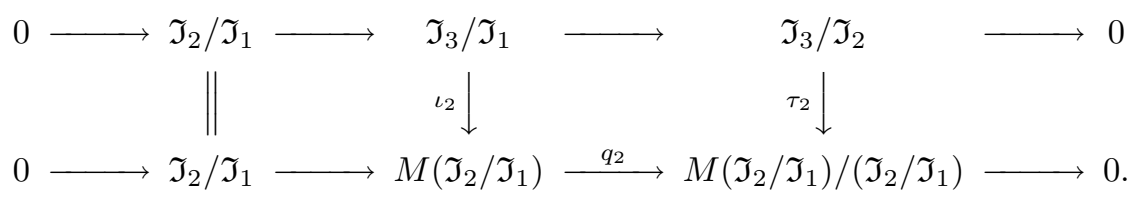

Note that $\mathfrak{I}_{2} / \mathfrak{I}_{1}$ is essential in $\mathfrak{I}_{3} / \mathfrak{I}_{1}$ since $\mathfrak{I}_{2}$ is essential in $\mathfrak{I}_{3}$. By the same reasoning as in the first case, we have

$$
\begin{aligned}
\mathfrak{I}_{3} / \mathfrak{I}_{1} & \cong M\left(\mathfrak{I}_{2} / \mathfrak{I}_{1}\right) \oplus_{q_{2}, \tau_{2}} \mathfrak{I}_{3} / \mathfrak{I}_{2} \\
& \cong \Gamma^{b}\left(X_{2},\{K\}\right) \oplus_{q_{2}, \tau_{2}} \mathfrak{I}_{3} / \mathfrak{I}_{2} \cong\left(\Gamma^{b}\left(X_{2},\{K\}\right) \otimes K\right) \oplus_{q_{2}, \tau_{2}} \mathfrak{I}_{3} / \mathfrak{I}_{2} .
\end{aligned}
$$

By using [9, Proposition 1.6] and [2, Proposition 3.3],

$$
\begin{aligned}
\operatorname{RR}\left(\mathfrak{I}_{3} / \mathfrak{I}_{1}\right) & \leq \max \left\{\operatorname{RR}\left(\Gamma^{b}\left(X_{2},\{K\}\right) \otimes K\right), \operatorname{RR}\left(\mathfrak{I}_{3} / \mathfrak{I}_{2}\right)\right\} \\
& \leq \max \left\{1, \operatorname{RR}\left(\mathfrak{I}_{3} / \mathfrak{I}_{2}\right)\right\}=1
\end{aligned}
$$


since $\mathfrak{I}_{3} / \mathfrak{I}_{2} \cong \lim _{\longrightarrow} C_{0}\left(U_{3 k}\right) \otimes K$ so that

$$
\left.\mathrm{RR}\left(\stackrel{\lim }{\longrightarrow} C_{0}\left(U_{3 k}\right) \otimes K\right) \leq \sup _{k} \operatorname{RR}\left(C_{0}\left(U_{3 k}\right) \otimes K\right)\right) \leq 1 .
$$

Furthermore, we have

$$
\begin{aligned}
\mathfrak{I}_{3} & \cong M\left(\mathfrak{I}_{1}\right) \oplus_{q_{1}, \tau_{1}} \mathfrak{I}_{3} / \mathfrak{I}_{1} \\
& \cong \Gamma^{b}\left(X_{1},\{K\}\right) \oplus_{q_{1}, \tau_{1}} \mathfrak{I}_{3} / \mathfrak{I}_{1} \cong\left(\Gamma^{b}\left(X_{1},\{K\}\right) \otimes K\right) \oplus_{q_{1}, \tau_{1}} \mathfrak{I}_{3} / \mathfrak{I}_{1} .
\end{aligned}
$$

Therefore, by [9, Proposition 1.6] and [2, Proposition 3.3] we obtain

$$
\begin{aligned}
\operatorname{RR}\left(\mathfrak{I}_{3}\right) & \leq \max \left\{\operatorname{RR}\left(\Gamma^{b}\left(X_{1},\{K\}\right) \otimes K\right), \operatorname{RR}\left(\mathfrak{I}_{3} / \mathfrak{I}_{1}\right)\right\} \\
& \leq \max \left\{1, \operatorname{RR}\left(\mathfrak{I}_{3} / \mathfrak{I}_{1}\right)\right\}=1 .
\end{aligned}
$$

For the general step, we use the following diagrams:

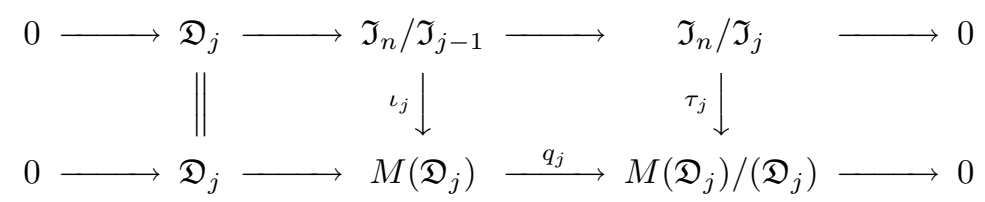

for $1 \leq j \leq n-1$, where $\mathfrak{D}_{j}=\mathfrak{I}_{j} / \mathfrak{I}_{j-1}$ and $\mathfrak{I}_{0}=\{0\}$. By the same reasoning as in the first case, we have

$$
\begin{aligned}
\mathfrak{I}_{n} / \mathfrak{I}_{j-1} & \cong M\left(\mathfrak{D}_{j}\right) \oplus_{q_{j}, \tau_{j}} \mathfrak{I}_{n} / \mathfrak{I}_{j} \\
& \cong \Gamma^{b}\left(X_{j},\{K\}\right) \oplus_{q_{j}, \tau_{j}} \mathfrak{I}_{n} / \mathfrak{I}_{j} \cong\left(\Gamma^{b}\left(X_{j},\{K\}\right) \otimes K\right) \oplus_{q_{j}, \tau_{j}} \mathfrak{I}_{n} / \mathfrak{I}_{j} .
\end{aligned}
$$

By [9, Proposition 1.6] and [2, Proposition 3.3] we obtain

$$
\begin{aligned}
\operatorname{RR}\left(\mathfrak{I}_{n} / \mathfrak{I}_{j-1}\right) & \leq \max \left\{\operatorname{RR}\left(\Gamma^{b}\left(X_{j},\{K\}\right) \otimes K\right), \operatorname{RR}\left(\mathfrak{I}_{n} / \mathfrak{I}_{j}\right)\right\} \\
& \leq \max \left\{1, \operatorname{RR}\left(\mathfrak{I}_{n} / \mathfrak{I}_{j}\right)\right\} .
\end{aligned}
$$

Using this inequality repeatedly for $j$ varying, we have $\operatorname{RR}\left(\mathfrak{I}_{n}\right) \leq 1$.

Since the union $\cup_{j} \mathfrak{I}_{j}$ is dense in $\mathfrak{A}$, we obtain $R R(\mathfrak{A}) \leq \sup _{j} R R\left(\mathfrak{I}_{j}\right) \leq 1$ as desired.

Remark. It is shown in Takai and the author [18, Proposition 3.1] that

$$
\operatorname{sr}(\mathfrak{A}) \leq 2
$$

for $\mathfrak{A}$ a separable $C^{*}$-algebra of type I which have no finite dimensional irreducible representations, where $\operatorname{sr}(\cdot)$ means the stable rank of $C^{*}$-algebras (see [12]). By definition, $\operatorname{sr}(\mathfrak{A}) \in\{1,2, \cdots, \infty\}$. However, their method is much different from the method in the proof above.

As an important application, we have 
Corollary 2.2. Let $G$ be a non-amenable $C C R$ locally compact group and $C_{r}^{*}(G)$ its reduced group $C^{*}$-algebra. Then

$$
\operatorname{RR}\left(C_{r}^{*}(G)\right) \leq 1 .
$$

Proof. It is known that if $G$ is a non-amenable locally compact group, then $C_{r}^{*}(G)$ has no finite dimensional irreducible representations (cf. [4, Chapter 18 and 18.9.5]).

Remark. It is shown in [13, Proposition 2.3] that

$$
\operatorname{sr}\left(C_{r}^{*}(G)\right) \leq 2
$$

for $G$ a non-amenable locally compact group of type I.

In particular, we obtain

Theorem 2.3. Let $G$ be a non-compact connected real semi-simple Lie group and $C_{r}^{*}(G)$ its reduced group $C^{*}$-algebra. Then

$$
\operatorname{RR}\left(C_{r}^{*}(G)\right)=1 .
$$

Proof. It is known that $G$ is CCR (cf. [4, Chapter 17 and 17.4.6]), and $G$ is nonamenable since it is non-compact. Thus, $\operatorname{RR}\left(C_{r}^{*}(G)\right) \leq 1$ by Corollary 2.2. When $G$ has real rank one as a Lie group via Iwasawa decomposition, it follows from the structure of $C_{r}^{*}(G)$ given by [13, Lemma 2.2] that $C_{r}^{*}(G)$ has a closed ideal of the form $C_{0}(R) \otimes K$. Since $C_{0}(R) \otimes K$ has no projections we have $\operatorname{RR}\left(C_{0}(R) \otimes K\right) \geq 1$. By [2, Proposition 3.3] we have $\operatorname{RR}\left(C_{0}(R) \otimes K\right) \leq 1$. Therefore, by [5, Theorem 1.4] we have $\operatorname{RR}\left(C_{r}^{*}(G)\right) \geq \operatorname{RR}\left(C_{0}(R) \otimes K\right)=1$. When $G$ has real rank more than one as a Lie group, it follows from the structure of $C_{r}^{*}(G)$ given by [13, Lemma 2.1] that $C_{r}^{*}(G)$ has a closed ideal of the form $C_{0}(X) \otimes K$ for $X$ a certain non-compact connected locally compact Hausdorff space. Hence, by the same argument as above we deduce $\operatorname{RR}\left(C_{r}^{*}(G)\right) \geq 1$.

Remark. It is shown in [13, Theorem 2.5] that

$$
\operatorname{sr}\left(C_{r}^{*}(G)\right)=\min \{2, \operatorname{rr}(G)\}
$$

for $G$ a non-compact connected real semi-simple Lie group, where $\operatorname{rr}(G)$ is the real rank of $G$.

Furthermore, we obtain

Theorem 2.4. Let $G$ be a non-compact connected real reductive Lie group and $C_{r}^{*}(G)$ its reduced group $C^{*}$-algebra. Then

$$
\operatorname{RR}\left(C_{r}^{*}(G)\right)=1 .
$$


Proof. Since $G$ is reductive, non-compact and connected, there exists a non-compact connected semi-simple quotient Lie group $S$ of $G$. In fact, $S \cong G / R$ for $R$ the radical of $G$ (i.e, the largest connected solvable Lie group in $G$ ). Then $C_{r}^{*}(S)$ is a quotient of $C_{r}^{*}(G)$ since $R$ is amenable. Thus, we obtain $\operatorname{RR}\left(C_{r}^{*}(G)\right) \geq \operatorname{RR}\left(C_{r}^{*}(S)\right)=1$ by [5, Theorem 1.4] and our Theorem 2.3. Since $G$ is CCR and non-amenable, we have $\operatorname{RR}\left(C_{r}^{*}(G)\right) \leq 1$ by Corollary 2.2 .

Remark. It is shown in [13, Theorem 3.1] ([15, Theorem 3.1]) that

$$
\operatorname{sr}\left(C_{r}^{*}(G)\right)=\min \left\{2, \max \left\{\operatorname{rr}([G, G]), \operatorname{dim} Z^{\wedge}+1\right\}\right\}
$$

for $G$ a non-compact connected real reductive Lie group, where $[G, G]$ is the commutator group of $G$, and $Z$ is the center of $G$ and $Z^{\wedge}$ is its dual group.

As another consequence of Theorem 2.1 we have

Corollary 2.5. Let $\mathfrak{A}$ be an inductive limit of $C C R C^{*}$-algebras which have no finite dimensional irreducible representations. Then

$$
\operatorname{RR}(\mathfrak{A}) \leq 1
$$

Proof. This follows from the property of the real rank for inductive limits of $C^{*}$-algebras (cf. [3, Proposition 3.1] and use its generalization).

On the other hand, we now recall that a $C^{*}$-algebra $\mathfrak{A}$ is $n$-homogeneous if for any irreducible representation $\pi$ of $\mathfrak{A}$, its image $\pi(\mathfrak{A})$ of $\mathfrak{A}$ is isomorphic to $M_{n}(C)$, and $\mathfrak{A}$ is $\infty$-homogeneous if $\pi(\mathfrak{A}) \cong K$ for any irreducible representation $\pi$ of $\mathfrak{A}$ (cf. [4]). Following the methods as in Theorem 2.1 we can obtain

Theorem 2.6. Let $\mathfrak{A}$ be a $C C R C^{*}$-algebra. Suppose that $\mathfrak{A}$ has no infinite dimensional irreducible representations. Then we have

$$
\operatorname{RR}(\mathfrak{A})=\sup _{j}\left\lceil\operatorname{dim} X_{j} /\left(2 n_{j}-1\right)\right\rceil
$$

for a composition series $\left\{\mathfrak{I}_{j}\right\}$ of $\mathfrak{A}$ such that subquotients $\mathfrak{I}_{j} / \mathfrak{I}_{j-1}$ are isomorphic to $C_{0}\left(X_{j}\right) \otimes M_{n_{j}}(C)$ for some $n_{j}$, where $\lceil x\rceil$ means the least integer $\geq x$.

Proof. We have treated the case for $\infty$-homogeneous $C^{*}$-algebras in Theorem 2.1. Using the notations as in the proof of Theorem 2.1 we deal with the case for subquotients with continuous trace to be $n$-homogeneous $C^{*}$-algebras. In fact, since $\mathfrak{A}$ is CCR, by the assumption we have a composition series $\left\{\mathfrak{I}_{j}\right\}$ of $\mathfrak{A}$ with such subquotients $\mathfrak{I}_{j} / \mathfrak{I}_{j-1}$. Thus, we replace the subquotients $\mathfrak{I}_{j} / \mathfrak{I}_{j-1}=\Gamma_{0}\left(X_{j},\{K\}\right)$ in the proof of Theorem 2.1 with $\Gamma_{0}\left(X_{j},\left\{M_{n_{j}}(C)\right\}\right)$ for some $n_{j}$. Furthermore, since each $\Gamma_{0}\left(X_{j},\left\{M_{n_{j}}(C)\right\}\right)$ is locally trivial, we may assume and replace that $\mathfrak{I}_{j} / \mathfrak{I}_{j-1}=C_{0}\left(X_{j}\right) \otimes M_{n_{j}}(C)$ (if necessary by transfinite induction for the subquotients). Then we have $M\left(\mathfrak{I}_{1}\right) \cong C^{b}\left(X_{1}\right) \otimes M_{n_{1}}(C) \cong C\left(\beta X_{1}\right) \otimes M_{n_{1}}(C)$, where 
$C^{b}\left(X_{1}\right)$ is the $C^{*}$-algebra of bounded continuous functions on $X_{1}$, and $\beta X_{1}$ is the Stone-Čech compactification of $X_{1}$ (cf. [1]). Thus, using [9, Proposition 1.6] and [2, Corollary 3.2] we obtain

$$
\begin{aligned}
\operatorname{RR}\left(\mathfrak{I}_{2}\right) & \leq \max \left\{\operatorname{RR}\left(M\left(\mathfrak{I}_{1}\right)\right), \operatorname{RR}\left(\mathfrak{I}_{2} / \mathfrak{I}_{1}\right)\right\} \\
& =\max \left\{\operatorname{RR}\left(C\left(\beta X_{1}\right) \otimes M_{n_{1}}(C)\right), \operatorname{RR}\left(C_{0}\left(X_{2}\right) \otimes M_{n_{2}}(C)\right)\right\} \\
& =\max \left\{\left\lceil\operatorname{dim} \beta X_{1} /\left(2 n_{1}-1\right)\right\rceil,\left\lceil\operatorname{dim} X_{2} /\left(2 n_{2}-1\right)\right\rceil\right\}
\end{aligned}
$$

and repeating this process above we obtain

$$
\mathrm{RR}(\mathfrak{A}) \leq \sup _{j}\left\lceil\operatorname{dim} X_{j} /\left(2 n_{j}-1\right)\right\rceil .
$$

On the other hand, by [5] we have

$$
\operatorname{RR}(\mathfrak{A}) \geq \operatorname{RR}\left(\mathfrak{I}_{j}\right) \geq \operatorname{RR}\left(\mathfrak{I}_{j} / \mathfrak{I}_{j-1}\right)=\left\lceil\operatorname{dim} X_{j} /\left(2 n_{j}-1\right)\right\rceil .
$$

Therefore, we obtain the conclusion as desired.

Remark. For any $C^{*}$-algebra $\mathfrak{A}$, we have

$$
\operatorname{RR}(\mathfrak{A}) \geq \sup _{1 \leq n<\infty}\left\lceil\operatorname{dim} X_{n} /(2 n-1)\right\rceil,
$$

where $X_{n}$ means the subspace of the spectrum of $\mathfrak{A}$ consisting of $n$-dimensional irreducible representations of $\mathfrak{A}$. Note that some or all $X_{n}$ may be empty. Moreover, by the same way we have

$$
\operatorname{sr}(\mathfrak{A}) \geq \sup _{1 \leq n<\infty}\left(1+\left\lceil\left[\operatorname{dim} X_{n} / 2\right] / n\right\rceil\right),
$$

where $[x]$ means the maximum integer $\leq x$. For a $C^{*}$-algebra $\mathfrak{A}$ of type $\mathrm{I}$, it is shown by [16, Corollary 2.8] that

$$
\operatorname{sr}(\mathfrak{A}) \leq \max \left\{2, \sup _{1 \leq n<\infty}\left(1+\left\lceil\left[\left(1+\operatorname{dim} X_{n}\right) / 2\right] / n\right\rceil\right)\right\} .
$$

As a general result containing Theorem 2.1 and Theorem 2.6 in part,

Theorem 2.7. Let $\mathfrak{A}$ be a CCR $C^{*}$-algebra. Suppose that $\mathfrak{A}$ is decomposed into the exact sequence:

$$
0 \rightarrow \mathfrak{I} \rightarrow \mathfrak{A} \rightarrow \mathfrak{D} \rightarrow 0,
$$

where $\mathfrak{I}$ is a CCR $C^{*}$-algebra that has no finite dimensional irreducible representations and $\mathfrak{D}$ is a CCR $C^{*}$-algebra that has no infinite dimensional irreducible representations. Then

$$
\operatorname{RR}(\mathfrak{D}) \leq \operatorname{RR}(\mathfrak{A}) \leq \max \{1, \operatorname{RR}(\mathfrak{D})\} .
$$


Namely, in other words,

$$
\sup _{j}\left\lceil\operatorname{dim} X_{j} /(2 j-1)\right\rceil \leq \mathrm{RR}(\mathfrak{A}) \leq \max \left\{1, \sup _{j}\left\lceil\operatorname{dim} X_{j} /(2 j-1)\right\rceil\right\},
$$

where $X_{j}$ are the subspaces of the spectrum of $\mathfrak{A}$ consisting of $j$-dimensional irreducible representations.

Remark. Note that quotients and closed ideals of CCR $C^{*}$-algebras are CCR. But extensions of CCR $C^{*}$-algebras by CCR $C^{*}$-algebras are not always CCR (cf. [4]). In fact, such extensions may not have Hausdorff spectrums.

To prove this theorem we need the following lemma :

Lemma 2.8. Let $\mathfrak{A}$ be a CCR $C^{*}$-algebra. Suppose that $\mathfrak{A}$ is decomposed into the exact sequence:

$$
0 \rightarrow \mathfrak{I} \rightarrow \mathfrak{A} \rightarrow \mathfrak{D} \rightarrow 0,
$$

where $\mathfrak{I}$ is an essential closed ideal of $\mathfrak{A}$ and is a continuous trace $C^{*}$-algebra that has no finite dimensional irreducible representations and $\mathfrak{D}$ is a CCR $C^{*}$-algebra. Then

$$
\operatorname{RR}(\mathfrak{A}) \leq \max \{1, \operatorname{RR}(\mathfrak{D})\} .
$$

Proof. We use the first step of the argument in the proof of Theorem 2.1. Namely, replace $\mathfrak{I}_{1}$ and $\mathfrak{I}_{2} / \mathfrak{I}_{1}$ with $\mathfrak{I}$ and $\mathfrak{D}$ respectively.

Remark. If a $C^{*}$-algebra $\mathfrak{A}$ has an $\infty$-homogeneous closed ideal $\mathfrak{I}$ with continuous trace, then it is shown by [10] that

$$
\operatorname{sr}(\mathfrak{A}) \leq \max \{2, \operatorname{sr}(\mathfrak{A} / \mathfrak{I})\} .
$$

Proof of Theorem 2.7. Since $\mathfrak{A}$ is CCR (and of type I), there exists an essential composition series $\left\{\mathfrak{I}_{j}\right\}$ of $\mathfrak{A}$ such that subquotients $\mathfrak{I}_{j} / \mathfrak{I}_{j-1}$ are of continuous trace and each $\mathfrak{I}_{j}$ is essential in $\mathfrak{I}_{j+1}$. For each $j$, we consider the following exact sequence:

$$
0 \rightarrow \mathfrak{I} \cap \mathfrak{I}_{j} \rightarrow \mathfrak{I}_{j} \rightarrow \mathfrak{D}_{j} \rightarrow 0,
$$

where the quotient $\mathfrak{D}_{j}$ corresponds to the subspace $\mathfrak{D}^{\wedge} \cap\left(\mathfrak{I}_{j}^{\wedge} \backslash\left(\mathfrak{I} \cap \mathfrak{I}_{j}\right)^{\wedge}\right)$, where $\mathfrak{B}^{\wedge}$ for a $C^{*}$-algebra $\mathfrak{B}$ means the spectrum of $\mathfrak{B}$ consisting of its irreducible representations up to unitary equivalence. Now set $\mathfrak{K}_{k}=\mathfrak{I} \cap \mathfrak{I}_{k}$ for $1 \leq k \leq j$ and $\mathfrak{K}_{0}=\{0\}$. Then we consider the following exact sequences:

$$
0 \rightarrow \mathfrak{K}_{k} / \mathfrak{K}_{k-1} \rightarrow \mathfrak{I}_{j} / \mathfrak{K}_{k-1} \rightarrow \mathfrak{I}_{j} / \mathfrak{K}_{k} \rightarrow 0
$$

for $1 \leq k \leq j$. Note that $\mathfrak{I}_{j} / \mathfrak{K}_{k-1}$ are CCR and $\mathfrak{K}_{k} / \mathfrak{K}_{k-1}$ are $\infty$-homogeneous continuous trace $C^{*}$-algebras. Hence, using the argument for the general step in the proof of Theorem 2.1 and Lemma 2.8 repeatedly we obtain

$$
\operatorname{RR}\left(\mathfrak{I}_{j} / \mathfrak{K}_{k-1}\right) \leq \max \left\{1, \operatorname{RR}\left(\mathfrak{I}_{j} / \mathfrak{K}_{k}\right)\right\}
$$


for $1 \leq k \leq j$. This implies that

$$
\operatorname{RR}\left(\mathfrak{I}_{j}\right) \leq \max \left\{1, \operatorname{RR}\left(\mathfrak{D}_{j}\right)\right\} \leq \max \{1, \operatorname{RR}(\mathfrak{D})\} .
$$

Note that $\mathfrak{D}_{j}$ are subquotients of $\mathfrak{D}$. Since the union of $\mathfrak{I}_{j}$ is dense in $\mathfrak{A}$, we have

$$
\operatorname{RR}(\mathfrak{A}) \leq \sup \operatorname{RR}\left(\mathfrak{I}_{j}\right) \leq \max \{1, \operatorname{RR}(\mathfrak{D})\}
$$

as desired.

As an important application, we obtain

Theorem 2.9. Let $G$ be a connected nilpotent Lie group and $C^{*}(G)$ its group $C^{*}$-algebra. Then

$$
\operatorname{RR}\left(C^{*}(G)\right)=\operatorname{dim} G_{1}^{\wedge},
$$

where $G_{1}^{\wedge}$ is the space of all 1-dimensional representations of $G$.

Proof. Since $C^{*}(G)$ (or $G$ ) is CCR and we have the following exact sequence:

$$
0 \rightarrow \mathfrak{I} \rightarrow C^{*}(G) \rightarrow C_{0}\left(G_{1}^{\wedge}\right) \rightarrow 0,
$$

where $\mathfrak{I}$ is a CCR $C^{*}$-algebra that has no finite dimensional irreducible representations (note that since $G$ is a solvable Lie group, the Lie's theorem tells us that $G$ (or $C^{*}(G)$ ) has no finite dimensional irreducible representations except 1-dimensional ones), we can use Theorem 2.7 so that

$$
\operatorname{RR}\left(C_{0}\left(G_{1}^{\wedge}\right)\right) \leq \operatorname{RR}\left(C^{*}(G)\right) \leq \max \left\{1, \operatorname{RR}\left(C_{0}\left(G_{1}^{\wedge}\right)\right)\right\} .
$$

By [3, Proposition 1.1] we have $\operatorname{RR}\left(C_{0}\left(G_{1}^{\wedge}\right)\right)=\operatorname{dim} G_{1}^{\wedge}$. Moreover, [14] implies that $\operatorname{dim} G_{1}^{\wedge}=0$ if and only if $G$ is isomorphic to the $k$-torus $T^{k}$. In this case we have $\operatorname{RR}\left(C^{*}(G)\right)=0$ since $C^{*}\left(T^{k}\right) \cong C_{0}\left(Z^{k}\right)$ by the Fourier transform and $C_{0}\left(Z^{k}\right) \cong$ $\oplus_{Z^{k}} C$ the $c_{0}$-direct sum of $C$. Furthermore, by [14] we have $\operatorname{dim} G_{1}^{\wedge}=1$ if and only if $G$ is isomorphic to either $R \times T^{k}$ or $R$. In this case we have $\operatorname{RR}\left(C^{*}(G)\right)=1$ since $C^{*}\left(R \times T^{k}\right) \cong C_{0}\left(R \times Z^{k}\right)$. If $\operatorname{dim} G_{1}^{\wedge} \geq 2$, then the inequalities obtained above imply the conclusion.

Remark. Note that the space $G_{1}^{\wedge}$ is homeomorphic to the dual group $(G /[G, G])^{\wedge}$ of the quotient connected abelian Lie group $G /[G, G]$ so that $G /[G, G] \cong R^{s} \times T^{t}$ and hence $G_{1}^{\wedge} \approx R^{s} \times Z^{t}$ for some $s, t \geq 0$. For $G$ a connected nilpotent Lie group, it is shown by [14] that

$$
\operatorname{sr}\left(C^{*}(G)\right)=\left[\operatorname{dim} G_{1}^{\wedge} / 2\right]+1 .
$$

This generalizes the case for $G$ a simply connected nilpotent Lie group by [17]. 


\section{References}

[1] C. A. Akemann, G. K. Pedersen and J. Tomiyama, Multipliers of $C^{*}$-algebras, J. Funct. Anal., 13(1973), 277-301.

[2] E. J. Beggs and D. E. Evans, The real rank of algebras of matrix valued functions, Internat. J. Math., 2(1991), 131-138.

[3] L. G. Brown and G. K. Pedersen, $C^{*}$-algebras of real rank zero, J. Funct. Anal., 99(1991), 131-149.

[4] J. Dixmier, $C^{*}$-algebras, North-Holland, Amsterdam, 1977.

[5] N. Elhage Hassan, Rang réel de certaines extensions, Proc. Amer. Math. Soc., 123(1995), 3067-3073.

[6] J. Hjelmborg and M. Rørdam, On stability of $C^{*}$-algebras, J. Funct. Anal., 155(1998), 153-170.

[7] E. Kaniuth, Group $C^{*}$-algebras of real rank zero or one, Proc. Amer. Math. Soc., 119(1993), 1347-1354.

[8] K. Nagami, Dimension Theory, Academic Press, New York-London, 1970.

[9] M. Nagisa, H. Osaka and N.C. Phillips, Ranks of algebras of continuous $C^{*}$-algebra valued functions, Canad. J. Math., 53(2001), 979-1030.

[10] V. Nistor, Stable rank for a certain class of type $I C^{*}$-algebras, J. Operator Theory, 17(1987), 365-373.

[11] G. K. Pedersen, $C^{*}$-Algebras and their Automorphism Groups, Academic Press, London-New York-San Francisco, 1979.

[12] M. A. Rieffel, Dimension and stable rank in the $K$-theory of $C^{*}$-algebras, Proc. London Math. Soc., 46(1983), 301-333.

[13] T. Sudo, Stable rank of the reduced $C^{*}$-algebras of non-amenable Lie groups of type I, Proc. Amer. Math. Soc., 125(1997), 3647-3654.

[14] T. Sudo, Dimension theory of group $C^{*}$-algebras of connected Lie groups of type I, J. Math. Soc. Japan, 52(2000), 583-590.

[15] T. Sudo, Survey on the rank and structure theory of group $C^{*}$-algebras of Lie groups, Ryukyu Math. J., 13(2000), 79-95.

[16] T. Sudo, Stable rank of $C^{*}$-algebras of type I, Linear Algebra Appl., 383(2004), 65-76.

[17] T. Sudo and H. Takai, Stable rank of the $C^{*}$-algebras of nilpotent Lie groups, Internat. J. Math., 6(1995), 439-446.

[18] T. Sudo and H. Takai, Stable rank of the $C^{*}$-algebras of solvable Lie groups of type I, J. Operator Theory, 38(1997), 67-86.

[19] N. E. Wegge-Olsen, K-theory and $C^{*}$-algebras, Oxford Univ. Press, Oxford-New YorkTokyo, 1993. 